\title{
CONSIDERAÇÕES SOBRE O ENSINO DO PACIENTE CIRÚRGICO
}

\author{
Márcia Maria Fontão Zago*
}

ZAGO, M.M.F. Consideraçōes sobre o ensino do paciente cinúrgico. Rev. Esc. Enf. USP., v.27, n.1, p. 67-71, abr. 1993.

O ensino do paciente cirúrgico tem sido considerado como uma estratégia de atuação do enfermeiro, com o objetivo de facilitar a recuperaçäo e minimizar as consequências que possam repercutir na qualidade de vida pós-cirúrgica. O objetivo deste estudo é apresentar consideraçòes sobre o ensino do paciente cirúrgico no periodo perioperatório.

UNITERMOS: Ensino de Paciente. Paciente Cinúrgico.

\section{INTRODUÇÄO}

A evolução técnico-científica das ciências biológicas expandiu as técnicas cirúrgicas, levando a um grande avanço dos conhecimentos sobre como os riscos cirúrgicos podem ser minimizados. Entretanto, a intervenção cirúrgica continua sendo considerada como um momento de crise para o indivíduo.

Os objetivos dos procedimentos cirúrgicos são vários: de diagnóstico, de tratamento cletivo, de prevenção, para aliviar sintomas, de cura. Todo ato cirúrgico, por menor que seja sua extensão, desencadeia uma série de reaçōes fisiológicas e psicológicas. A ruptura da integridade dos tecidos, a perda hidro-eletrolítica, a lesão dos órgãos, provocam uma série de reações endócrinas que repercutem em todo o organismo. O sucesso de uma cirurgia depende, assim, do estado de saúde do indivíduo no período anterior a cirurgia, e de como ele se comporta durante a fase pós cirúrgica (BELEND; PASSOS, 1979).

O paciente cirúrgico está exposto às mesmas fontes de tensão que outros pacientes hospitalizados: o afastamento dos familiares e de suas atividades diárias, o ambiente desconhecido. Entretanto, algumas são peculiares: o trauma fisiológico, a dor, o conhecimento do diagnóstico c do objetivo da cirurgia, a anestesia, e as consequências para o seu estilo de vida, imagem corporal e auto-imagem. Por mais bem planejada que scja uma cirurgia, os riscos scmpre existem, e são eles que provocam, no indivíduo, um comportamento caracterizado pela ansiedade (ELHART et al, 1983).

O desenvolvimento das técnicas cirúrgicas tem propiciado a melhora do nível de saúde $\mathrm{cm}$ relação a função orgânica; algumas destas técnicas, porém, acarretam dificuldades psicossociais para o paciente. A realização de estomas (traqueostomia, colostomia), a retirada de órgãos (mastectomia), e mesmo a cirurgia cardíaca, são procedimentos que têm aumentado em frequência, com

Professora Assistemte, Doutoranda do Departamento de Enfermagem Geral e Especializada da Escola de Enfermagem de Ribeirăo Preto -USP. 
consequente diminuição da morbidade e mortalidade, mas com repercussóes para a qualidade de vida dos pacientes. Esses pacientes, $\mathrm{cm}$ um curto período de tempo, têm scu estilo de vida, sua imagem corporal, sua auto-estima alterados. E muitas vezes precisam aprender habilidades para que possam exccutar seu auto-cuidado (REDMAN, 1976; WILSON-BARNETT, 1983).

Esse estudo, então, tem por objetivo tecer consideração sobre o ensino do paciente cirúrg ico c as diferenças do processo no perioperatório.

\section{O ENSINO DO PACIENTE CIRÚRGICO}

O enfermeiro, como membro da equipe cirúrgica, também tem procurado acompanhar a evoluçāo técnico-científico da cirurgia, aprofundando os conhecimentos adquiridos, de modo a atuar efetivamente na assistência ao paciente cirúrgico. Uma dessas formas de atuação é representada pelo ensino de pacientes, que consiste na ação educativa do enfermeiro com o paciente, promovendo o desenvolvimento de atitudes e estratégias novas, frente a um problema específico de saúde (ZAGO, 1990). É considerado uma estratégia de atuação possível, ocorrendo por tempo determinado, como também, uma açāo paralcla à sistematizaçāo dos cuidados de enfermagem (REDMAN, 1976; WILSON-BARNETT, 1983).

Com o desenvolvimento sócio-cultural, o paciente passou a ser valorizado como um ser humano com capacidades c responsabilidades por manter sua saúde, e não mero recipiente das ações médicas. O processo de ensino de pacientes é, entāo, cncarado como um meio pelo qual o paciente pode adquirir conhecimentos e habilidades, ser encorajado a participar do seu tratamento, tomando decisōes, assumindo responsabilidades.

Os dados obtidos em cstudos sobre as consequências da cirurgia para o paciente, demonstram que o ensino desses pacientes é vantajoso para o desenvolvimento da sua independência $\mathrm{em}$ relação à equipe de saúde, manutenção da saúdc, diminuição da gravidade da doença c redução da ansiedade. Esses resultados têm repercussões na redução do tempo de internação, no custo do tratamento para o indivíduo c para a instituição (SMITHERMAN, 1984; ATKINSON; MURRAY, 1989).

\section{AS FASES DO ENSINO DO PACIENTE CIRÚRGICO}

Considero quc o ensino do paciente cirúrgico deva ser descnvolvido em duas fases críticas, no pré c no pós-operatório. No intra-operatório, fase do ato cirúrgico propriamente dito, o paciente permanece sob a ação anestésica, impedindo-o de interagir com o ambiente.

O pré-operatório, período que se estende desde a internação do paciente até o seu encaminhamento para a sala de operações, é um momento difícil para o paciente, quando a sua ansicdade pode atingir níve is máximos. Para acentuar ainda mais essa ansiedade, realizam-se procedimentos preparatórios que 
visam favorecer as condi-çōes fisiológicas e aumentar a probalidade de que o organismo responda adequadamente ao trauma cirúrgico, como: exames de sangue, Raio-X, tricotomia, enema, punção venosa; procedimentos que se concentram todos num curto período de tempo, pois, em geral, a internação ocorre vinte e quatro horas antes da cirurgia.

Além do preparo do paciente quanto aos aspectos somáticos, a atuação sobre o estressc psicológico é fundamental para a rápida recuperaçāo. O ensino de pacientes, no pré-operatório, influência as fases posteriores da cirurgia, levando à redução do tempo de hospitalização e de complicações pós-cirúrgicas, neutralizando os sentimentos de desespero e insegurança que agravam a ansiedade (BRILLHART; STEWART, 1989; HUSSEY; GILLILAND, 1989; RUZICK, 1989; SMITH,1989).

O conteúdo desse ensino visa esclarecer as dúvidas dos pacientes, acrescentar informaçōes, a diantar possíveis situações a serem experienciadas. Essa instrução deverá ser um processo com explicações e demonstrações. Só assim, o paciente poderá desenvolver capacidades que the permitam compreender as situações, procurar alternativas que minimizem sua ansiedade (WILSONBARNETT, 1983). FAULKNER (1985) esclarece que o nível de informações a serem oferecidas no pré-operatório, depende das necessidades do paciente e da sua capacidade de assimilar a informação. Contudo, informar não promove necessariamente segurança. A oportunidade de dialogar, de expor seus medos, é que poderá reduzir a tensão e encorajar o paciente a participar. Em geral, é preconizado que depende do nível de ansiedade do paciente, as informações devam ser gerais, pois, informações excessivas podem acentuar a ansiedade.

O período pós-operatório inicia quando o paciente deixa a sala de opcração e é encaminhado ao Centro de Recuperação. Após a recuperação anestésica, e tendo padrōes fisiológicos equilibrados, o paciente retorna ao seu leito, na enfermaria (BELAND; PASSOS, 1979). É o período mais longo, podendo durar dias ou meses, dependendo da intervenção cirúrg ica realizada.

$\mathrm{Na}$ cnfermaria, com o passar do tempo, o paciente volta a integrar-se no ambiente. Iniciando com uma grande dependência em relação à equipe de enfermagem, aos poucos pode reassumir suas atividades. É nesse momento que as informaçōes e conhecimentos adquiridos no pré-operatório poderão ser utilizados pelo paciente em seu próprio benefício.

Com o restabelecimento da capacidade cognitiva, podem-se acrescentar novos conhecimentos, apresentar fatos e soluções para a alta hospitalar. Para muitos enfermeiros, a alta hospitalar é sinônimo de sucesso e missão cumprida (CALDERA, 1980). Entretanto, é realista pensar que nenhuma pessoa que deixa o hospital estará totalmente independente; há uma dependência relativa (FAULKER, 1985). Tem-se descrito que grande porcentagem de pacientes retornam ao hospital com recidivas de problemas que poderiam ter sido prevenidos (CALDERA, 1980). Segundo CLAUSEN (1984), a alta hospitalar 
é considerada como o processo de transferência da responsabilidade do cuidado do paciente para a família ou outros profissionais de saúde.

AGUILLAR (1990) comenta sobre a alta precoce, estratégia utilizada para a racionalização de recursos na saúde, como uma causa de má adaptação pós-cirúrg ica, domiciliar, do paciente. A autora entrevistou pacientes cirúrgicos, após a alta hospitalar, advindos de um hospital-escola e constatou que $100 \%$ das pessoas manifestaram alguma dificuldade: $64 \% \mathrm{~cm}$ relação a mudança no estilo de vida, $60 \%$ de dependência em outras pessoas, $56 \% \mathrm{~cm}$ necessidade de orientação e $52 \% \mathrm{~cm}$ habilidades para o auto-cuidado. A autora conclui que aqueles pacientes receberam alta hospitalar despreparados $c$ desinformados.

\section{CONSIDERAÇÖES GERAIS}

O ensino de pacientes cirúrgicos ć considerado insubstituível, no processo cirúrgico (BILLE, 1981 ; WILSON-BARNETT, 1983). Não se pode menosprezar as suas consequências fisiológicas, econômicas, sociais e psicológicas. Também não se pode ignorar a importância da participação dos familiares, já que cles terão destaque no cuidado ao paciente, após a alta hospitalar.

Entretanto, o ensino do paciente cirúrgico só será efetivo se for adequado às neccssidades de cada paciente, ao tempo de hospitalização, às características individuais de aprendizagem, ao tipo c consequências da intervençào cirúrgica. Como todo processo, há princípios que promovem a efetivação. A adequação do contcúdo, os métodos, a prontidão do paciente e a importância da informação recebida pelo paciente não podem ser negligenciados pelos enfermeiros cducadores (GRADY et al, 1988; STEELE; RUZICHI, 1987).

ZAGO, M.M.F. Considerations about the teaching of surgical patient. Rev. Esc. Enf. USP.,, v.27, n.1, p. 67-71, apr. 1993.

Teaching of surgical patients is an established approach of nursing care aimed at promoting the recovery of the patient and lessening the untoward consequences wich may affect post-surgery life. The requirements about teaching surgical patient are discussed in this study.

UNITERMS: Patient teaching. Surgical patient.

\section{Referências Bibliográficas}

AGULI.AR, O.M. Aalta do paciente cirúrgiceno contexto do sistema de saúde. Ri beirão Preto, 1990. 104p. Tese (Doutorado) - Escola de Enfermagem de Ribeirāo Preto, Universidade de São Paulo.

ATHINSON, L.D.; MURRAY, M.E. Eundamentos de enfermagem: introduçāo ao processo de enfermagem. Rio de Janeiro, Guanabara Koogan, 1989. cap. 9, p. 97-114: O processo de ensino-aprendizagem.

BELAND, I.L.; PASSOS, J.Y. Enfermagem clínica: aspectos fisio-patológicos e psicossociais. Sāo Paulo, EPU/EDUSP, 1979. v. 3. 
BILLE, D.A. Practical approaches to patient teaching. Boston, Little-Brown, 1981.

BRILHART, B.; STEW ART, A. Education as the key to reabilitation. Nurs. Cin. North Am. v.24, n.3, p.675-80, 1989.

CALDERA, R. Exploration of the cffect of educational livel on the nurse's attitude toward discharge teaching. J. Nurs Educ., v. 19, n. 8, p. 24-32, 1980.

Cl.AUSEN, C. Staft RN: a discharge planner for every pati ent. Nurs Manage., v. 15, n. 11, p. 58-61, 1984.

EL .HART, D. et al. Princípios cientificos de enfermagem. Lisboa, Livros Técnicos e Científicos, 1983.

FAULKNER, A. Nursing: a creative approach. London, Bailliere-Tindall, 1985.

GRADY, K.L. et al. Patient perœpion of cardiovascular surgical patient education. Heart Lung, v. 17, n. 4 , p. 349-55, 1988.

HUSSFY, L.C.; GILLII.AND, K. Compliance, low literacy and locus of control. Nurs. Clin. North Am, v. 24, n. 3, p. 605-11, 1989.

REDMAN, B.R. The process of patient teaching in nursing. 3 ed. St. Louis, Mosby, 1976.

RUZICK, D.A. Reallistically meeting the educational needs of hospitalized acute and short-stay patients. Nurs. Cin. North Am., v. 24, n. 3, p. 583-7, 1989.

SMITH, C.E. Overview of patient education. Nurs. Clin. North $\Delta$ m., v. 24, n. 3, p. 583-7, 1989.

SMITHERMAN, C. Nursing actions for health promotion. 4 ed. Philadelphia, Davis, 1984. Cap. 4, p. 121-56: The teaching process.

STELI.E, J..; RUZICK, D. An evaluation of the effectiveness of cardiac teaching during hospitalization. Hearl Lung, v. 16, n. 3, p. 306-11, 1987.

WII SON-BARNET, J. Recent advance in nursing: patient teaching. Fdinburgh, Churchill Livingstone, 1983.

ZAGO, M. M.F. Plano de ensino para o preparo da alta médica do paciente laringectomizado. Ri beirāo Preto, 1990. 145p. Dissertação (Mestrado) - Escola de Enfermagem de Ribeirāo Preto, Universidade de Sāo Paulo. 